\title{
Galactic tide and local stellar perturbations on the Oort cloud: creation of interstellar comets ${ }^{\star} \star \star$
}

\author{
S. Torres, M. X. Cai, A. G. A. Brown, and S. P. Zwart \\ Leiden Observatory, Leiden University, PO Box 9513, 2300 RA Leiden, The Netherlands \\ e-mail: storres@strw.leidenuniv.nl
}

Received 21 February 2019 / Accepted 24 July 2019

\begin{abstract}
Comets in the Oort cloud evolve under the influence of internal and external perturbations, such as giant planets, stellar passages, and the Galactic gravitational tidal field. We aim to study the dynamical evolution of the comets in the Oort cloud, accounting for the perturbation of the Galactic tidal field and passing stars. We base our study on three main approaches; analytic, observational, and numerical. We first construct an analytical model of stellar encounters. We find that individual perturbations do not modify the dynamics of the comets in the cloud unless very close $(<0.5 \mathrm{pc})$ encounters occur. Using proper motions, parallaxes, and radial velocities from Gaia DR2 and combining them with the radial velocities from other surveys, we then construct an astrometric catalogue of the 14659 stars that are within $50 \mathrm{pc}$ of the Sun. For all these stars we calculate the time and distance of closest approach to the Sun. We find that the cumulative effect of relatively distant $(\leq 1 \mathrm{pc})$ passing stars can perturb the comets in the Oort cloud. Finally, we study the dynamical evolution of the comets in the Oort cloud under the influence of multiple stellar encounters from stars that pass within $2.5 \mathrm{pc}$ of the Sun and the Galactic tidal field over $\pm 10 \mathrm{Myr}$. We use the Astrophysical Multipurpose Software Environment (AMUSE), and the GPU-accelerated direct N-body code ABIE. We considered two models for the Oort cloud, compact $(a \leq 0.25 \mathrm{pc})$ and extended $(a \leq 0.5 \mathrm{pc})$. We find that the cumulative effect of stellar encounters is the major perturber of the Oort cloud for a compact configuration while for the extended configuration the Galactic tidal field is the major perturber. In both cases the cumulative effect of distant stellar encounters together with the Galactic tidal field raises the semi-major axis of $\sim 1.1 \%$ of the comets at the edge of the Oort cloud up to interstellar regions $(a>0.5 \mathrm{pc})$ over the $20 \mathrm{Myr}$ period considered. This leads to the creation of transitional interstellar comets (TICs), which might become interstellar objects due to external perturbations. This raises the question of the formation, evolution, and current status of the Oort cloud as well as the existence of a "cloud" of objects in the interstellar space that might overlap with our Oort cloud, when considering that other planetary systems should undergo similar processes leading to the ejection of comets.
\end{abstract}

Key words. Oort Cloud - comets: general - stars: kinematics and dynamics - solar neighborhood

\section{Introduction}

The outer region of the solar system is populated by a large number of planetesimals. Further away, more than $1000 \mathrm{AU}$ from the Sun, and almost extending to the nearest stars, is the Oort cloud. Its existence was proposed in the late 1950s by the Dutch astronomer Jan Hendrik Oort, who realised that long-term comets (with orbital semi-major axes $a>40 \mathrm{AU}$ ) bound to the Sun must come from an area well beyond Neptune. Oort (1950) pointed out that a spike in the distribution of $1 / a$ of the longperiod comets with $a>10^{4} \mathrm{AU}$, and isotropic inclinations in $\cos i, \omega$, and $\Omega$, would argue for the existence of a reservoir of objects in quasi-spherical symmetry surrounding the solar system. The Oort cloud has remained unobserved to date.

There have been numerous studies aimed at trying to explain the formation, evolution, and structure of the Oort cloud, mostly through numerical simulation (e.g. Hills 1981; Heisler \& Tremaine 1986; Duncan et al. 1987; Weissman 1996; Wiegert \& Tremaine 1999; García-Sánchez et al. 1999; Dybczyński 2002; Levison et al. 2004; Dones et al. 2004; Morbidelli 2008;

\footnotetext{
${ }^{\star}$ The movie associated to Fig. 11 is available at http://wwW. aanda.org

$\star \star$ The full Table 3 is also available at the CDS via anonymous ftp to cdsarc.u-strasbg. fr (130.79.128.5) or via http://cdsarc. u-strasbg.fr/viz-bin/cat/J/A+A/629/A139
}

Duncan 2008; Brasser et al. 2006; Fouchard et al. 2006; Kaib \& Quinn 2008; Brasser \& Morbidelli 2013; Shannon et al. 2014; Dones et al. 2015). There is general agreement on some properties of the Oort cloud, in particular that it is composed of the residual planetesimals after the planet formation epoch. The Oort cloud is divided into two regions: the inner Oort cloud is usually reserved for comets with semi-major axes $a<20000 \mathrm{AU}$ and is invisible unless there is a comet shower. The outer Oort cloud refers to comets with semi-major axes $a>20000 \mathrm{AU}$ (e.g. Dones et al. 2015). Its shape is thought to be nearly spherical and limited at $0.5 \mathrm{pc}$ mainly by the influence of the Galactic tidal field and stellar flybys (e.g. Heisler \& Tremaine 1986). The Oort cloud is thought to contain around $10^{12}$ objects with a total mass of $\sim 3 \times 10^{25} \mathrm{~kg}$ (e.g. Morbidelli 2008). However, these estimations are highly uncertain. The above-mentioned studies also concluded that in order for long-period comets to still exist today they need to be replenished. Otherwise they would have been depleted on a timescale much shorter than the lifetime of the solar system.

The orbits of the comets in the Oort cloud form a frozen record of the evolution of the solar system and preserve the memory of its birth environment (Portegies Zwart \& Jílková 2015; Martínez-Barbosa et al. 2016; Fouchard et al. 2011, 2018). External perturbations such as Galactic tides, stellar flybys, and molecular clouds play an important role in the understanding of 
the formation and evolution of the Oort cloud and Oort cloudlike structures in other planetary systems (see e.g. Veras et al. 2013, 2014). Passing stars can perturb the comets, changing their perihelion distances much more than they change the overall size of the orbit, changing the cometary trajectories and injecting the comets into the inner solar system (Morbidelli 2008; Duncan 2008). The outer Oort cloud has been affected quite substantially by external influences. Not only by passing stars in the parental cluster of the Sun but also by occasional relatively close encounters that have occurred after the Sun has left its birth cluster (Jílková et al. 2016). Jílková et al. (2015) pointed out that the planetesimals Sedna and 2008PV113 belong to the inner Oort cloud and that they may have been captured during an encounter with another star in the birth cluster of the Sun. This star is conjectured to have passed the solar system within about $340 \mathrm{AU}$ and would have deposited approximately 1400 other planetesimals together with the two currently known objects in this family. The orbital characteristics of these objects share similar properties which can be used to reconstruct the encounter.

Close encounters with the solar system have been studied by a number of authors (e.g. Rickman 1976; Matthews 1994; Weissman 1996; Dehnen \& Binney 1998; García-Sánchez et al. 1999; Levison et al. 2004; Jimenez-Torres et al. 2011; BailerJones 2015; Dybczyński \& Berski 2015; Higuchi \& Kokubo 2015; Feng \& Bailer-Jones 2015; Berski \& Dybczyński 2016). Most of them calculated the closest encounters with the solar system within $\pm 10 \mathrm{Myr}$ using the astrometric data of the stars in the solar neighbourhood $(<50 \mathrm{pc})$ provided by HIPPARCOS mission (Perryman et al. 1997). They find that the closest approach $(\sim 0.3 \mathrm{pc})$ in the future $(\sim 1.3 \mathrm{Myr}$ from the present) will be with the star HIP 8982 (GJ 710), which will cause minor changes in the perihelion distance of the comets. The most recent close stellar encounter was with the so-called Scholz's star $\left(M_{\star} \simeq\right.$ $0.15 M_{\odot}$ at a distance of $0.25_{-0.07}^{+0.11} \mathrm{pc}$, Scholz 2014, Mamajek et al. 2015). All of the studies cited above were limited by the observational data due to the incompleteness of the HIPPARCOS survey.

The first data release (Gaia DR1) of the European Space Agency's Gaia mission (Gaia Collaboration 2016a,b) opened a new window for understanding the Milky Way. In the particular case of the solar system, Gaia detected nearly all of the local star systems within $50 \mathrm{pc}$ of the Sun (compared to the $20 \%$ detected by HIPPARCOS). Using Gaia DR1, several authors (Berski \& Dybczyński 2016; Bobylev \& Bajkova 2017; Torres et al. 2018; Bailer-Jones 2018) re-computed the orbit of the closest stars to the Sun. They found new stars and new parameters for some of the very well known encounters, such as GJ 710, which gets closer $(0.064 \mathrm{pc})$ based on the Gaia DR1 data. The recent second Gaia data release - Gaia DR2 (Gaia Collaboration 2018 ) - provided 7.2 million radial velocities. This provided an opportunity to find new and more accurately characterised stellar encounters. Using Gaia DR2, Bailer-Jones et al. (2018) found 693 new stars with closest-encounter distances within $5 \mathrm{pc}$ and 15 Myrs from now; accounting for the incompleteness they also re-calculate the present rate of encounters, which within $\sim 1 \mathrm{pc}$ of the Sun is estimated to be $20 \pm 2 \mathrm{Myr}^{-1}$. From HipPARCOS data García-Sánchez et al. (1999) derived $11.7 \pm 1.3 \mathrm{Myr}^{-1}$ within $\sim 1$ pc and Martínez-Barbosa et al. (2017) employed simulations to derive rates of 21,39 , and $63 \mathrm{Myr}^{-1}$ within $\sim 2 \mathrm{pc}$ for three different scenarios (orbital migration from the Milky Way inner disk, migration from the outer disk, and no migration, respectively).

We aim to obtain a conservative estimate of the combined effects of stellar encounters and the Galactic tidal field on the
Oort cloud, by only considering the encounters from stars listed in Gaia DR2 within \pm 10 Myr from the present. The latter sample is incomplete and thus provides a lower limit on the effects of passing stars. In Sect. 2 we present a simple analytical model for stellar encounters and discuss the cumulative effect of passing stars on the Oort cloud, using the impulse approximation. In Sect. 3 we present a catalogue of nearby stars and we calculate the effect of individuals encounters with stars within $2.5 \mathrm{pc}$ of the Sun. In Sect. 4 we present a numerical model for multiple stellar encounters and study the dynamical evolution of a simulated Oort cloud after the interaction with the nearby stars and the Galactic tidal field. Finally, in Sect. 5 we present our summary and conclusions.

\section{Model for stellar encounters}

The estimated extent of the Oort cloud is $\sim 0.5$ pc (Oort 1950; Dones et al. 2015), which means that the orbital velocity of bodies in the Oort cloud is limited to $0.13 \mathrm{~km} \mathrm{~s}^{-1}$. This implies that comets at the edge of the Oort cloud are barely bound to the Sun and thus the condition for a comet ejection due to an external perturbation, $\Delta v_{\perp}>v_{\text {esc }}$ (where $v_{\text {esc }}$ is the escape velocity) is easily met. The Galactic tidal field is the most important perturbation to the outer Oort cloud at large distances (Heisler \& Tremaine 1986). However, close encounters with stars also play an important role in the evolution of the Oort cloud.

\subsection{Analytic model}

A simple analytical model of stellar encounters can help us to better understand the effect of passing stars on the Oort cloud. To construct such a model, we followed the works of Rickman (1976), García-Sánchez et al. (2001), Rickman et al. (2004, 2008), and Martínez-Barbosa et al. (2017). We first compiled data for the mass, velocity dispersion, and the space density of the stars in the solar neighbourhood for 13 spectral types, as in Table 8 in García-Sánchez et al. (2001). The mass of the stars corresponding to the spectral types B0V to M5V was taken from the data compiled by Mamajeck $\left(2018\right.$, priv. comm.) ${ }^{1}$ (see also Pecaut \& Mamajek 2013). While the mean value for white dwarfs (WD) was taken from Jiménez-Esteban et al. (2018). The peculiar velocity of the Sun $\left(v_{\odot}\right)$ and the velocity dispersion of the stars $\left(v_{*}\right)$ were taken from Rickman et al. (2008). The space density of spectral types A to K and Giants was obtained from Bovy (2017). For B and M type stars the values were obtained from Rickman et al. (2008), and for the WD from Jiménez-Esteban et al. (2018). The compiled data are shown in Table 1.

We consider the effect of the stars with different masses $\left(M_{*}\right)$ and spectral types in the solar neighbourhood on the comets in the Oort cloud. We assume that the stars move on a straight line trajectory, and with a constant velocity relative to the Sun $\left(v_{*}\right)$. For high stellar velocities, we can assume that the comet is at rest during the stellar passage. Using the impulse approximation (Oort 1950; Rickman 1976), we then calculate the change of the velocity $\left(\Delta V_{\perp}\right)$ imparted to a comet in the Oort cloud due to a random stellar encounter by integrating the perpendicular force generated by each passing star:

$\Delta V_{\perp} \approx \frac{2 G M_{*}}{v_{*}}\left[\frac{\boldsymbol{r}_{c}}{r_{\mathrm{c}}^{2}}-\frac{\boldsymbol{r}_{\odot}}{r_{\odot}^{2}}\right]$,

where $r_{\mathrm{c}}$ and $r_{\odot}$ correspond to the vectors from the comet and the Sun to the point of closest approach of the star (assuming that

\footnotetext{
1 http://www.pas.rochester.edu/ emamajek/EEM_dwarf_ UBVIJHK_colors_Teff.txt
} 
Table 1. Stellar parameters.

\begin{tabular}{cccccc}
\hline \hline S.T & $M_{*}\left(M_{\odot}\right)$ & $v_{*}\left(\mathrm{~km} \mathrm{~s}^{-1}\right)$ & $v_{\odot}\left(\mathrm{km} \mathrm{s}^{-1}\right)$ & $v_{\text {enc }}\left(\mathrm{km} \mathrm{s}^{-1}\right)$ & $\rho_{*}\left(10^{-3} \mathrm{pc}^{-3}\right)$ \\
\hline B0V & 15 & 14.7 & 18.6 & 24.6 & 0.06 \\
A0V & 2.3 & 19.7 & 17.1 & 27.5 & 0.26 \\
A5V & 1.85 & 23.7 & 13.7 & 29.3 & 0.34 \\
F0V & 1.59 & 29.1 & 17.1 & 36.5 & 0.61 \\
F5V & 1.33 & 36.2 & 17.1 & 43.6 & 1.51 \\
G0V & 1.08 & 37.4 & 26.4 & 49.8 & 1.61 \\
G5V & 0.98 & 39.2 & 23.9 & 49.6 & 1.73 \\
K0V & 0.87 & 34.1 & 19.8 & 42.6 & 4.21 \\
K5V & 0.68 & 43.4 & 25.0 & 54.3 & 5.26 \\
M0V & 0.55 & 42.7 & 17.3 & 50.0 & 8.72 \\
M5V & 0.16 & 41.8 & 23.3 & 51.8 & 41.55 \\
WD & 0.6 & 63.4 & 38.3 & 80.2 & 4.9 \\
Giants & 2.2 & 41.0 & 21.0 & 49.7 & 3.9 \\
\hline
\end{tabular}

Notes. Columns represent the spectral type of the stars followed by their mass, velocity dispersion, and the peculiar velocity of the Sun with respect to each spectral type. The relative velocity of the encounter within the Sun-comet system and the star is shown in Col. 5 . The number density of stars in the solar neighbourhood is shown in Col. 6.

the comet has not been deflected by the gravity of the star). If we consider $r$ the heliocentric distance of the comet and we assume that the distance of the encounter is large enough compared to the distance Sun-comet, we can approximate Eq. (1) with:

$\Delta V_{\perp} \propto \frac{M_{*} r}{v_{*} r_{\odot}^{2}}$.

For the case of a very close encounter with the comet, Eq. (2) can be approximated as

$\Delta V_{\perp} \propto \frac{M_{*}}{v_{*} r_{\mathrm{c}}}$

It is important to stress that the impulse approximation is based on a number of simplifying assumptions, and therefore it should be used for statistical analysis only. For our propose, it gives us a general idea of the effect of the different stars in the solar neighbourhood on the comets in the Oort cloud.

Following Rickman (1976) we can calculate the frequency of the stellar encounters using

$f=\pi r_{*}^{2} v_{\text {enc }} \rho_{*}$,

where $r_{*}$ is the distance of the encounter, $v_{\text {enc }}=\sqrt{v_{\odot}^{2}+v_{*}^{2}}$ is the relative velocity of the Sun and a random passing star $\left(v_{\odot}\right.$ represents the peculiar velocity of the Sun, and $v_{*}$ the velocity dispersion of the parent population of the passing star), and $\rho_{*}$ is the number density of stars of a given spectral type in the solar neighbourhood. Equation (4) can be used to determine the number of stars passing by within a sphere of radius $r_{\mathrm{s}}$ centred on the Sun or a random comet (assuming that stars of the solar neighbourhood are uniformly distributed at any time and the stellar velocities relative to the Sun are constant, Rickman 1976):

$N_{*}=r_{\mathrm{s}}^{2} f t$.

\subsection{Perturbations on the Oort cloud}

Using Eq. (2) and the values in Table 1, we calculated the frequency of the stars passing within a distance $r_{*}$ from the Oort cloud. We find that the total frequency of stars passing
Table 2. Analytical model for stellar encounters for an encounter distance of $1 \mathrm{pc}$.

\begin{tabular}{cccc}
\hline \hline S.T & $f_{*}^{1 \mathrm{pc}}\left(\mathrm{Myr}^{-1}\right)$ & $\Delta V_{\perp, *}^{1 \mathrm{pc}}\left(\mathrm{km} \mathrm{s}^{-1}\right)$ & $N_{*}^{1 \mathrm{pc}}$ \\
\hline B0V & 0.005 & $8.77 \mathrm{e}-03$ & 4.742 \\
A0V & 0.023 & $1.005 \mathrm{e}-03$ & 22.973 \\
A5V & 0.032 & $6.716 \mathrm{e}-04$ & 32.007 \\
F0V & 0.072 & $4.701 \mathrm{e}-04$ & 71.536 \\
F5V & 0.212 & $3.161 \mathrm{e}-04$ & 211.527 \\
G0V & 0.258 & $2.485 \mathrm{e}-04$ & 257.608 \\
G5V & 0.276 & $2.151 \mathrm{e}-04$ & 275.696 \\
K0V & 0.576 & $2.195 \mathrm{e}-04$ & 576.229 \\
K5V & 0.918 & $1.348 \mathrm{e}-04$ & 917.675 \\
M0V & 1.401 & $1.108 \mathrm{e}-04$ & 1400.844 \\
M5V & 6.915 & $3.293 \mathrm{e}-05$ & 6915.189 \\
WD & 1.263 & $8.143 \mathrm{e}-05$ & 1262.623 \\
Giants & 0.623 & $4.617 \mathrm{e}-04$ & 622.765 \\
\hline
\end{tabular}

Notes. The spectral type is shown in Col. 1, the frequency of the stellar encounters is shown in Col. 2, the change in the velocity of a comet due to an interaction with a star is shown in Col. 3, and the total number of stars entering a sphere of radius $1 \mathrm{pc}$ around the Sun-comet system over a time interval of $1 \mathrm{Gyr}$ is shown in Col. 4 .

within $1 \mathrm{pc}$ is around $12.5 \mathrm{Myr}^{-1}$ (see Table 2). Following the same method, García-Sánchez et al. (1999) found a lower value $\left(11.7 \mathrm{Myr}^{-1}\right)$. The main difference with our result is due to the updated values for the mass and density of the stars used in this work. The most probable perturber of the Oort cloud is the low mass, high-relative-velocity stars.

Using Eq. (2), in Fig. 1 we show the change of the velocity of a comet due to an encounter with a star for different spectral types and as a function of the distance of the encounter for an interval of $0.1-2.5 \mathrm{pc}$. The lower distance corresponds to the inner Oort cloud, while the larger distance corresponds to the limit where a passing star can start perturbing a comet at the edge of the cloud. In the rest of this work we refer to the latter distance as the critical radius.

As shown in Fig. 1 the change induced by a single encounter is relatively small. Massive stars are effective in exciting the 


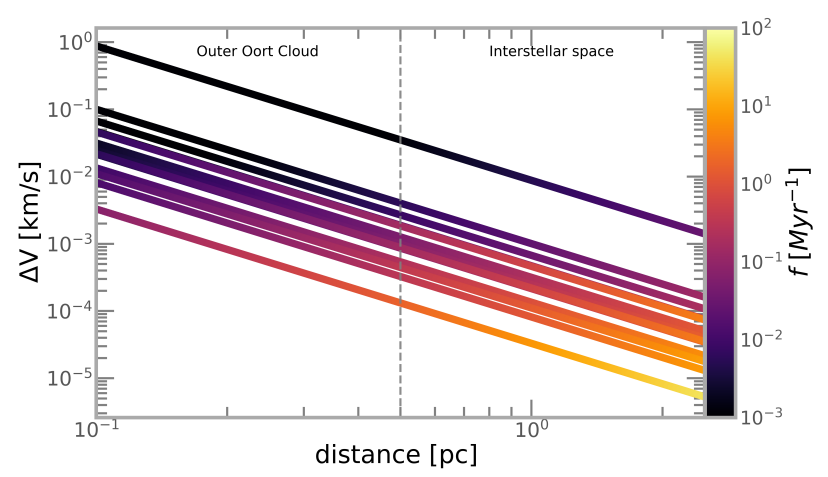

Fig. 1. Change of the velocity of a comet due to random stellar encounters for the 13 stellar spectral types listed in Table 1 as a function of the encounter distance. The colour coding of the lines represents the frequency of stellar encounters as a function of the distance of the encounter for the corresponding types of stars. The lines represent the different spectral types in the order listed in Table 1. The mass of the stars decreases from the top to the bottom lines.

object in the Oort cloud, but they are rare. Low-mass stars are very common, but their effect on the orbits of a comet is small. However the number of stars encountering the solar system increases over time.

The model presented in this section is based on a number of simplifying assumptions. Specifically, the impulse approximation provides a quick but inaccurate estimate of the effect of a random passing star on a comet in the Oort cloud. As we show in Table 2 the effect of individual stars is relative small. However, considering the frequency and the number of stars approaching the Sun to within $1 \mathrm{pc}$ over $1 \mathrm{Gyr}$, their cumulative effect might change the structure and dynamics of the Oort cloud. In order to have a better understanding of the evolution of the Oort cloud it is necessary to employ a detailed numerical model which accounts for the effects of the Galactic tidal field and stellar distribution of stars around the Sun. In Sect. 3 we present a list of nearby stars within $50 \mathrm{pc}$ of the Sun for which Gaia DR2 astrometry and radial velocities (including from other surveys) are available. This provides us with accurate kinematic information on nearby stars that could influence the Oort cloud in the recent past or near future. In Sect. 4 we use numerical simulations to analyse their effect on the comets in the Oort cloud and estimate their cumulative effect over $\pm 10 \mathrm{Myr}$, including the effect of the Galactic tidal field.

\section{Close encounters with the solar system}

Our knowledge of close stellar encounters in the recent past or near future has been limited by the availability of precise and accurate astrometry and radial velocities for the nearby stars. The Gaia mission has considerably increased the availability of astrometric and radial velocity data for the closest stars, even if about $20 \%$ of the stars with high proper motions are not listed in Gaia DR2 and those tend to be close to the Sun (Bailer-Jones et al. 2018).

\subsection{Observational model}

To construct the list of stars within $50 \mathrm{pc}$ of the Sun for which the encounter parameters (closest approach distance, velocity, and time) can be calculated we used the data from the Gaia DR2 catalogue. To increase the number of stars for which radial velocity information is available we cross-matched Gaia DR2 with the
Table 3. Overview of the catalogue of stars within $50 \mathrm{pc}$ of the Sun for which encounter parameters were calculated.

\begin{tabular}{lc}
\hline \hline Input catalogue(s) & $\begin{array}{c}\text { No. Stars } \\
\leq 50 \mathrm{pc}\end{array}$ \\
\hline Gaia DR2 & 10744 \\
Gaia DR2 + RAVE DR5 & 2356 \\
Gaia DR2 + GALAH DR2 & 11 \\
Gaia DR2 + LAMOST DR3 & 307 \\
Gaia DR2 + APOGEE DR14 & 1092 \\
Gaia DR2 + XHIP & 149 \\
\hline Total & 14659
\end{tabular}

Notes. The full catalogue is available at the CDS and can be download from: https://home.strw.leidenuniv.nl/ storres/ \#Research

following catalogues: RAVE-DR5 (Kunder et al. 2017), GALAH DR2 (Buder et al. 2018), LAMOST DR3 (Zhao et al. 2012), APOGEE DR14 (Abolfathi et al. 2018), and XHIP (Anderson \& Francis 2012). We selected only stars with relative uncertainty on the parallax $(\varpi)$ smaller than $20 \%$, such that $1 / \varpi$ is a good estimator of the distance to the stars. Following Lindegren et al. (2018) we further filtered the list of stars according to

$u^{2}<1.44 \times \max [1, \exp (-0.4(G-19.5))]$,

and

$1.0+0.015\left(G_{\mathrm{BP}}-G_{\mathrm{RP}}\right)^{2}<E<1.3+0.06\left(G_{\mathrm{BP}}-G_{\mathrm{RP}}\right)^{2}$,

where $G, G_{\mathrm{BP}}$, and $G_{\mathrm{RP}}$ correspond to the photometric measurements, covering a wavelength from the near-ultraviolet to the near-infrared for the $G$ passband, 330 to $680 \mathrm{~nm}$, and 630 to $1050 \mathrm{~nm}$ for $G_{\mathrm{BP}}$, and $G_{\mathrm{RP}}$, respectively. The $u=\left(\chi^{2} / v\right)^{1 / 2}$ corresponds to the unit weight error, and $E$ is the flux excess factor. This filter selects sources with high-quality astrometry and weeds out stars which appear to be nearby because of spuriously high values of the parallax (see Appendix $\mathrm{C}$ in Lindegren et al. 2018). The resulting catalogue contains 14659 stars within $50 \mathrm{pc}$ of the Sun (Table 3).

For the selected stars we estimated the distance, time, and velocity of closest approach using the linear approximation method of Matthews (1994) in the formulation presented in Bailer-Jones (2015):

$$
\begin{aligned}
& v_{\mathrm{tot}}=\sqrt{v_{\mathrm{T}}^{2}+v_{\mathrm{rad}}^{2}}, \\
& t_{\mathrm{ph}}=-\frac{c v_{\mathrm{rad}}}{\varpi v_{\mathrm{tot}}^{2}}, \\
& d_{\mathrm{ph}}=\frac{10^{3}}{\varpi} \frac{v_{\mathrm{T}}}{v_{\mathrm{tot}}}
\end{aligned}
$$

where $v_{\mathrm{T}}=4.74\left[\left(\mu_{\alpha *}{ }^{2}+\mu_{\delta}^{2}\right)^{0.5} / \varpi\right]$ is the transverse velocity, $v_{\text {rad }}$ is the radial velocity of the star, $\varpi$ is the parallax, $c=$ $10^{3} \mathrm{pc} \mathrm{km}^{-1} \mathrm{yr}^{-1}$, and the subscript "ph" stands for perihelion. We estimated the mass of the stars using the effective temperature provided in Gaia DR2 (Andrae et al. 2018) and linearly interpolating in the tables in Mamajeck (2018, priv. comm) and Pecaut \& Mamajek (2013).

Of the 14659 stars within $50 \mathrm{pc}$ there are 31 that pass within $2.5 \mathrm{pc}$ of the Sun (Fig. 2, big dots) over a period of $20 \mathrm{Myr}$ centred on the present (i.e. $10 \mathrm{Myr}$ in the past and $10 \mathrm{Myr}$ in the 


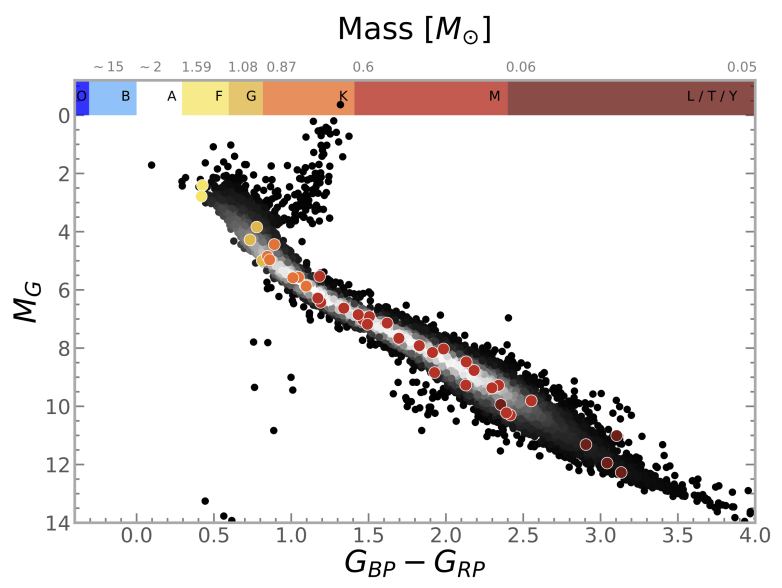

Fig. 2. Observational HR diagram of the nearby stars contained in the catalogue summarised in Table 3 . The big dots represent the stars within $2.5 \mathrm{pc}$ of the Sun colour coded according to their spectral type. The density map shows all the stars in our sample.

future). Figure 2 shows the observational Hertzsprung-Russell diagram of our sample. In Fig. 3 we show the closest approach distance and time of the stars in our sample with respect to the Sun. The limited distance range of the stars under study only allows us to find very close encounters within \pm 3 Myr.

In Fig. 3 the large dots show the distribution of the stars passing within $2.5 \mathrm{pc}$ and those tend to be the major perturber of the Oort cloud (referred to as "Gaia stars" below). The closest encounter with the solar system is GJ 710 which will penetrate deep inside the inner Oort cloud. As shown in Fig. 2 most of the closest encounters involve $M$ dwarfs, with a considerable fraction of solar type stars. This implies that the effect of a single encounter with the Oort cloud will be minimal, mainly due to the low mass of the perturber and its high velocity with respect to the Sun.

Bailer-Jones et al. (2018) find 62 new stellar encounters, which partially overlap with our list. Their list of encounters is larger than ours mostly because they did not apply the strict filtering on the astrometric quality of the Gaia DR2 data employed in this work. For stars appearing in both studies we find similar results.

We stress that the sample of the closest stars presented here is incomplete. The observational incompleteness is evident in the decrease in encounter frequency as one moves away from the present epoch in time. A complete census of stellar encounters requires all the stars within a certain distance to be identified. The main limitations in using the Gaia survey for finding the closest encounters are the survey magnitude limit, which prevents the identification of encounters with faint low-mass stars, and the lack of radial velocities. The Gaia DR2 radial velocity survey is limited to effective temperatures in the range $\sim 3550-6900 \mathrm{~K}$ and to stars brighter than $G=14$ mag (see e.g. Bailer-Jones et al. 2018). An additional limitation is that some of the brightest stars in the sky are missing from the Gaia DR2 catalogue (Gaia Collaboration 2018).

A detailed study correcting for incompleteness in Gaia DR2 was carried out by Bailer-Jones et al. (2018). These latter authors constructed a completeness map (Fig. 12, Bailer-Jones et al. 2018), interpreted as the probability of detecting a given close encounter in the Gaia DR2 sample. They found that only $15 \%$ of the encounters within $5 \mathrm{pc}$ in a period of $5 \mathrm{Myr}$ have been identified. Using this result, the authors used a simulated Milky
Way galaxy to infer the encounter rate averaged over $5 \mathrm{Myr}$, in the past and future. They found that the encounter rate of stellar encounters within $1 \mathrm{pc}$ is $20 \pm 2 \mathrm{Myr}^{-1}$.

\subsection{Stellar encounters with the Solar System}

In Sect. 3.1 we employed a simple method to estimate the perihelion distances and times for stars approaching the Sun by assuming the stars follow a uniform motion along straight lines with respect to the Sun (see also Bailer-Jones 2015). We now seek a better estimation of the perihelion distance through the joint integration of the orbits of the Sun and the stars that are predicted to approach to within $2.5 \mathrm{pc}$ (Table 4) backwards and forwards in time for $10 \mathrm{Myr}$. We first transformed the astrometric and radial velocity data into galactocentric Cartesian frame using Astropy (Astropy Collaboration 2018). We adopted the position of the Sun and the local circular velocity parameters from Reid et al. (2014): $Z_{\odot}=27 \mathrm{pc}, R_{\odot}=8.34 \mathrm{kpc}$, and $V_{c, \odot}=240 \mathrm{~km} \mathrm{~s}^{-1}$; while the peculiar velocity of the Sun was adopted from Schönrich et al. (2010): $\left(U_{\odot}, V_{\odot}, W_{\odot}\right)=$ $(11.1,12.24,7.25) \mathrm{km} \mathrm{s}^{-1}$. We used the Gala (Price-Whelan 2017) package to perform the orbital integration. The Milky Way potential used is described by an analytic axisymmetric model which contains a spherical nucleus and bulge (Hernquist 1990), a Miyamoto-Nagai disk (Miyamoto \& Nagai 1975; Bovy 2015), and a spherical Navarro-Frenk-White (NFW) dark matter halo (Navarro et al. 1996).

To account for the observational uncertainties we sample the astrometric and radial velocity observables for each star, taking the full covariance matrix into account. For each star, $10^{6}$ samples of the astrometry and radial velocity are drawn and for each of these the above described orbit integration is carried out. The end result is a sampling of the distribution of possible perihelion distances and times. This distribution obtained through Monte Carlo sampling is then treated as the probability density function (PDF) of the encounter parameters. The shape of the confidence regions is mainly affected by the relative errors on parallax and radial velocity. The relative error in the proper motion likewise affects the shape of the confidence regions around the mean. Figures 4-6 show the resulting PDFs.

In Fig. 4 we show the distribution of time and distance of closest approach for the time interval $\pm 3 \mathrm{Myr}$ from the present.The closest encounter found is, as expected, the very well known case of GJ 710. In Fig. 5 we show the distribution of the total relative velocity and distance of closest approach. Most stars in our sample have high velocities $\left(20-80 \mathrm{~km} \mathrm{~s}^{-1}\right)$ meaning that their effect on the Oort cloud is small. Figure 6 shows the distribution of the time and relative velocity of closest approach, showing a triangular shape with a peak toward high velocities and the present time. This is a selection effect caused by our limitation of the total studied sample to stars that are currently within $50 \mathrm{pc}$ of the Sun (this means that very fast-moving stars that would approach the Sun far in the past or the future are currently not in the $50 \mathrm{pc}$ volume).

We calculate the effect on a comet of a passing star that approaches to within $2.5 \mathrm{pc}$ (Table 4), using the impulse approximation (Eq. (2)). We find that the change in the velocity of a comet is relatively small (in the order of $10^{-3}-10^{-4} \mathrm{~km} \mathrm{~s}^{-1}$, Table 4). The exception is for the passage of GJ 710, which causes a velocity change of $\sim 0.13 \mathrm{~km} \mathrm{~s}^{-1}$, creating an important perturbation in the inner Oort cloud. Overall if only individual encounters are considered the Oort cloud comets barely feel the effect of passing stars. The impulse approximation is based on a number of simplifying assumptions, but this approach gives us a 


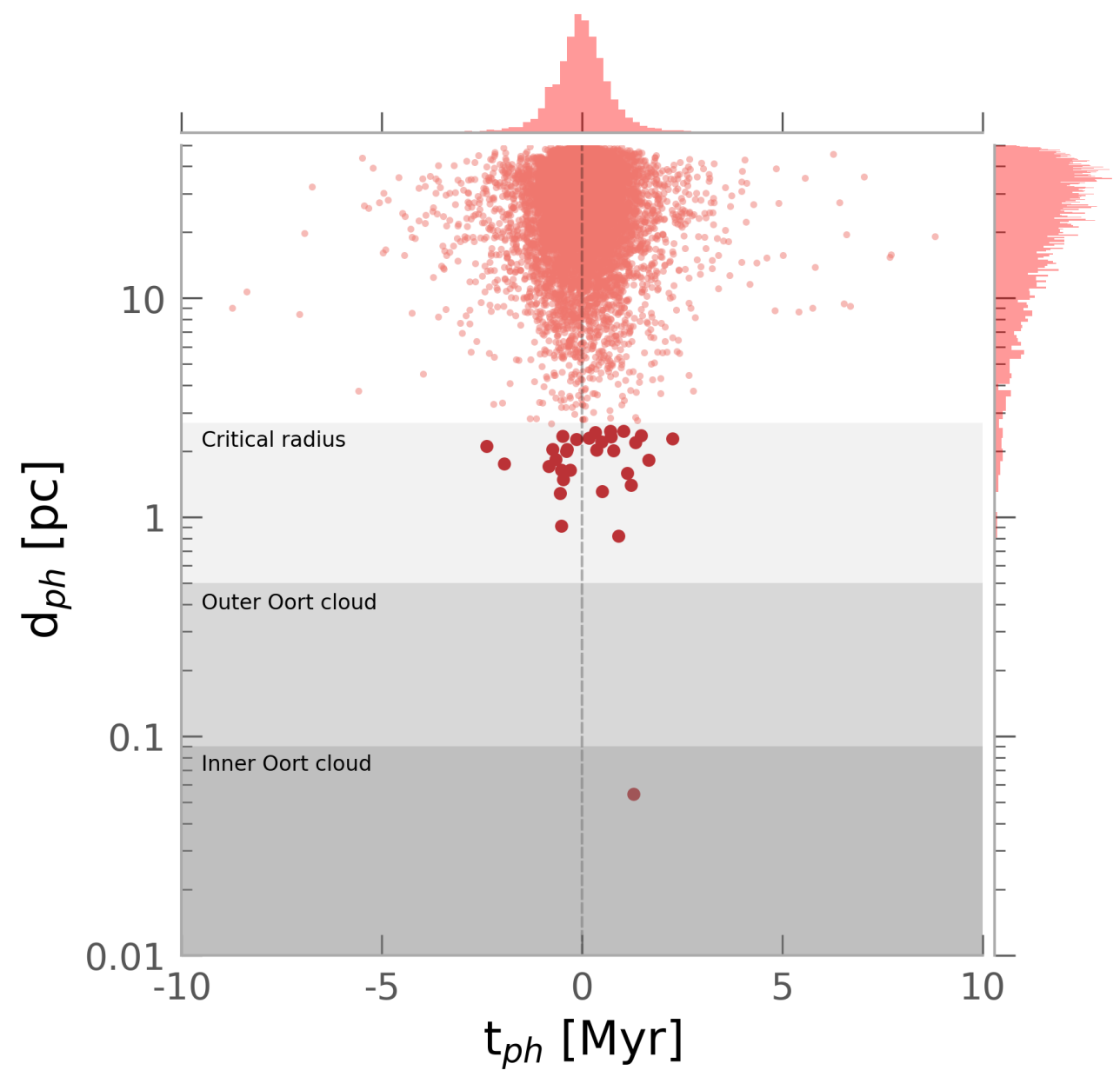

Fig. 3. Closest approach distance vs. closest approach time. The shaded areas represent the critical radius within which stars can affect an object in the Oort cloud, and the boundaries of the outer and inner Oort cloud, respectively. The big dots correspond to those stars that pass within $2.5 \mathrm{pc}$ of the Sun.

general panorama of the individual effect of the nearby stars on a comet in the Oort cloud. In order to quantify the global effect of passing stars, it is necessary to integrate their orbits backwards and forward in time (see Sect. 3.1). Such a scenario is shown in the third row of Fig. 9, Sect. 4.1. The cumulative effect of nearby stars is strong enough to lift the perihelion of $\sim 0.38 \%$ of the objects in the Oort cloud (Fig. 9, third row). Particularly the effect of GJ 710 is strong (see also discussion in Sect. 4.1 and Fig. 8), but encounters within $\sim 1$ pc also have an important contribution.

\subsection{The case of GJ 710/HIP 89825}

For decades GJ 710 has been pointed out as the major future perturber of the Oort cloud. The first calculations using HIPPARCOS catalogue led to an encounter distance of $0.33 \mathrm{pc}, 1.38 \mathrm{Myr}$ from the present time (see e.g. García-Sánchez et al. 2001; Torres et al. 2018). Using Gaia DR2, Torres et al. (2018) pointed out that the encounter distance is even smaller, at $0.062 \mathrm{pc}, 1.35 \mathrm{Myr}$ from today (see also Berski \& Dybczyński 2016; Bobylev \& Bajkova 2017; Bailer-Jones 2018). With the data from Gaia DR2 in hand, the distance and time of closest approach have again slightly decreased to $0.054 \mathrm{pc}$ and $1.28 \mathrm{Myr}$ (Table 3, 5). de la Fuente Marcos \& de la Fuente Marcos (2018) and Bailer-Jones et al. (2018) found similar but slightly discrepant results. The small discrepancy in the various results is mainly due to the orbit integration method and the Galactic potential used in their calculations, considering that the input data are the same.

A comparison of the results obtained for GJ 710 from Hipparcos, Gaia DR1, and Gaia DR2 data is shown in Fig. 7. Calculations were performed following the method described in Sect. 3.1, using the astrometric data described in Table 5. The discrepancy between HIPPARCOS and Gaia is due to the difference in the value of the astrometric parameters and radial velocity. This results in a shift in the perihelion distance of GJ 710. Using different parameters for the Galactic potential will also lead to slightly different values (see e.g. Bailer-Jones et al. 2018). We note that the time of perihelion is more uncertain for the Gaia DR2 data, which is caused by the larger uncertainty in the radial velocity. Following the method described in Sects. 3.2 and 4, we investigated the effect of GJ 710 on a simulated Oort cloud (see also discussion in Sect. 4.1 and the first row in Fig. 9). The perturbation because of GJ 710 lifts the semi-major axis of the comets within the region between $\sim 10000$ and $100000 \mathrm{AU}$ (see also discussion in Sect. 4.1 and Fig. 8), creating $\sim 0.01 \%$ hyperbolic objects, while $\sim 0.30 \%$ of the comets gain a semimajor axis beyond the edge of the Oort cloud ( $a>100000 \mathrm{AU})$.

\section{Dynamical evolution of the Oort cloud}

As pointed out by Heisler \& Tremaine (1986) the Galactic tidal field is a major contributor to Oort cloud perturbations at large 
Table 4. Stars predicted to approach the Sun within $2.5 \mathrm{pc}$ over the $\pm 10 \mathrm{Myr}$ from today.

\begin{tabular}{|c|c|c|c|c|c|c|c|c|c|}
\hline Gaia DR2 ID & $t_{\mathrm{ph}}(\mathrm{Myr})$ & $t_{\text {sample }}$ & $d_{\mathrm{ph}}(\mathrm{pc})$ & $d_{\text {sample }}$ & $v_{\text {tot }}\left(\mathrm{km} \mathrm{s}^{-1}\right)$ & $v_{\text {sample }}$ & $T_{\text {eff }}(\mathrm{K})$ & Mass $\left(M_{\odot}\right)$ & $\Delta v_{\perp}\left(\mathrm{km} \mathrm{s}^{-1}\right)$ \\
\hline 4270814637616488064 & 1.282 & {$[1.123,1.488]$} & 0.054 & {$[0.006,0.107]$} & 14.525 & {$[12.515,16.564]$} & 4116 & 0.654 & $1.305 \mathrm{e}-01$ \\
\hline 553219967007245312 & 1.670 & {$[1.595,1.742]$} & 1.824 & {$[1.714,1.952]$} & 24.163 & {$[23.187,25.284]$} & 5175 & 0.851 & $9.106 \mathrm{e}-05$ \\
\hline 258179971749627776 & 0.365 & {$[0.352,0.378]$} & 2.028 & {$[1.955,2.107]$} & 78.377 & {$[75.746,81.470]$} & 4507 & 0.717 & $1.915 \mathrm{e}-05$ \\
\hline 4575928186606190336 & 0.479 & {$[0.462,0.500]$} & 2.210 & {$[2.126,2.307]$} & 51.102 & {$[48.983,52.973]$} & 3795 & 0.559 & $1.925 \mathrm{e}-05$ \\
\hline 3240424426786618624 & -0.552 & {$[-0.572,-0.534]$} & 1.284 & {$[1.214,1.368]$} & 3.029 & {$[80.325,85.666]$} & 3836 & 0.580 & $3.647 \mathrm{e}-05$ \\
\hline 4795598309045006208 & -0.739 & {$[-0.757,-0.723]$} & 2.037 & {$[1.985,2.091]$} & 32.470 & {$[31.698,33.194]$} & 5343 & 0.901 & $5.755 \mathrm{e}-05$ \\
\hline 3274130814728561792 & -2.390 & {$[-2.645,-2.158]$} & 2.105 & {$[1.834,2.447]$} & 19.171 & {$[17.362,21.165]$} & 4471 & 0.715 & $7.240 \mathrm{e}-05$ \\
\hline 981375326780564608 & 0.508 & {$[0.476,0.548]$} & 1.314 & {$[1.229,1.422]$} & 53.595 & {$[49.613,57.211]$} & 3875 & 0.601 & $5.589 \mathrm{e}-05$ \\
\hline 6684504722300935680 & -0.465 & {$[-0.495,-0.436]$} & 1.488 & {$[1.400,1.587]$} & 3.104 & {$[40.449,45.973]$} & 3619 & 0.494 & -05 \\
\hline 4430238051199001216 & 0.167 & {$[0.165,0.171]$} & 2.295 & {$[2.225,2.379]$} & & {$[66.901,68.396]$} & 6017 & 28 & e-05 \\
\hline 2417069815934357248 & 2.253 & {$[2.049,2.486]$} & 2.280 & {$[2.068,2.531]$} & & {$[12.746,15.408]$} & 4613 & 0.741 & $35 \mathrm{e}-05$ \\
\hline 3089711447388931584 & -0.133 & {$[-0.139,-0.127]$} & 2.273 & {$[2.164,2.389]$} & & {$[60.457,66.818]$} & 3820 & 0.571 & $1.497 \mathrm{e}-05$ \\
\hline 3339921875389105152 & -0.516 & {$[-0.544,-0.485]$} & 1.639 & {$[1.541,1.732]$} & 1.455 & {$[20.317,22.843]$} & 410 & & $6 e-05$ \\
\hline 1134618591670426112 & 0.728 & {$[0.713,0.742]$} & 2.331 & {$[2.273,2.394]$} & & {$[62.639,65.223]$} & & & $1.950 \mathrm{e}-05$ \\
\hline 5861048509766415616 & -0.297 & {$[-0.304,-0.290]$} & 1.643 & {$[1.601,1.682]$} & & {$[57.962,60.702]$} & 37 & & $5 e-05$ \\
\hline 6819998 & 1.134 & {$[1.066,1.209]$} & 1.585 & {$[1.483,1.697]$} & & {$[14.795,16.794]$} & 3956 & 0.615 & e-04 \\
\hline 3260079227925564160 & 0.910 & {$[0.851,0.968]$} & 0.823 & {$[0.754,0.905]$} & 395 & {$[31.465,35.704]$} & 3998 & 0.629 & $2.395 \mathrm{e}-04$ \\
\hline 2648914040357320576 & 1.473 & {$[1.412,1.544]$} & 2.367 & {$[2.257,2.502]$} & 13.235 & {$[12.616,13.823]$} & 5630 & & $1.133 \mathrm{e}-04$ \\
\hline 3972130276695660288 & -0.511 & {$[-0.572,-0.458]$} & 0.912 & {$[0.818,1.025]$} & 31.845 & {$[28.459,35.551]$} & 3980 & 0.623 & $2.025 \mathrm{e}-04$ \\
\hline 2118161219075485824 & 0.779 & {$[0.743,0.816]$} & 2.016 & {$[1.908,2.126]$} & & {$[53.650,58.906]$} & 4122 & & $2.466 \mathrm{e}-05$ \\
\hline 1392610405193517952 & 0.702 & {$[0.586,0.8$} & 2.468 & {$[2.067,3.095]$} & 64. & {$[51.343,77.613]$} & 50 & & e- 05 \\
\hline 2089889682751105536 & 1.041 & {$[0.997,1.090]$} & 2.478 & {$[2.346,2.633]$} & 46.407 & {$[44.351,48.454]$} & 3965 & 0.618 & $1.866 \mathrm{e}-05$ \\
\hline 2272191085754928768 & 0.335 & {$[0.331,0.338]$} & 2.436 & {$[2.401,2.469]$} & 76.760 & {$[75.892,77.614]$} & 5859 & 1.060 & $2.003 \mathrm{e}-05$ \\
\hline 4758877919212831104 & -0.395 & {$[-0.405,-0.385]$} & 2.000 & {$[1.952,2.050]$} & 31.680 & {$[30.850,32.497]$} & 4893 & 0.788 & $5.352 \mathrm{e}-05$ \\
\hline 4839132097557586560 & -0.828 & {$[-0.856,-0.803]$} & 1.713 & {$[1.624,1.815]$} & 46.116 & {$[44.687,47.522]$} & 3865 & 0.597 & $3.798 \mathrm{e}-05$ \\
\hline 5076269164798852864 & -0.479 & {$[-0.503,-0.456]$} & 2.347 & {$[2.063,2.673]$} & & {$[49.277,51.577]$} & 4837 & 0.781 & $2.418 \mathrm{e}-05$ \\
\hline 4546557031272743680 & 1.216 & {$[1.163,1.272]$} & 1.398 & {$[1.311,1.490]$} & 35.688 & {$[34.137,37.245]$} & 4305 & 0.695 & $8.573 \mathrm{e}-05$ \\
\hline 875071278432954240 & 1.331 & {$[1.250,1.413]$} & 2.198 & {$[2.057,2.351]$} & 16.389 & {$[15.429,17.455]$} & 5714 & 0.998 & $1.085 \mathrm{e}-04$ \\
\hline 2924339469735490560 & -1.951 & {$[-2.039,-1.862]$} & 1.751 & {$[1.646,1.864]$} & 14.766 & {$[14.128,15.488]$} & 5743 & 1.009 & $1.918 \mathrm{e}-04$ \\
\hline 3371908043029299840 & -0.372 & {$[-0.379,-0.366]$} & 2.041 & {$[1.994,2.085]$} & 82.969 & {$[81.685,84.203]$} & 4330 & 0.700 & $1.742 \mathrm{e}-05$ \\
\hline 3369088315397965056 & -0.656 & {$[-0.670,-0.643]$} & 1.839 & {$[1.788,1.894]$} & 40.659 & {$[39.870,41.541]$} & 6020 & 1.130 & $7.066 \mathrm{e}-05$ \\
\hline
\end{tabular}

Notes. The columns represent the Gaia DR2 ID, the time, distance and velocity at the perihelion with its respective confidence interval. These are followed by the effective temperature listed in Gaia DR2, the estimated mass of the star, and the change in the velocity due to the encounter with the Sun for each star.

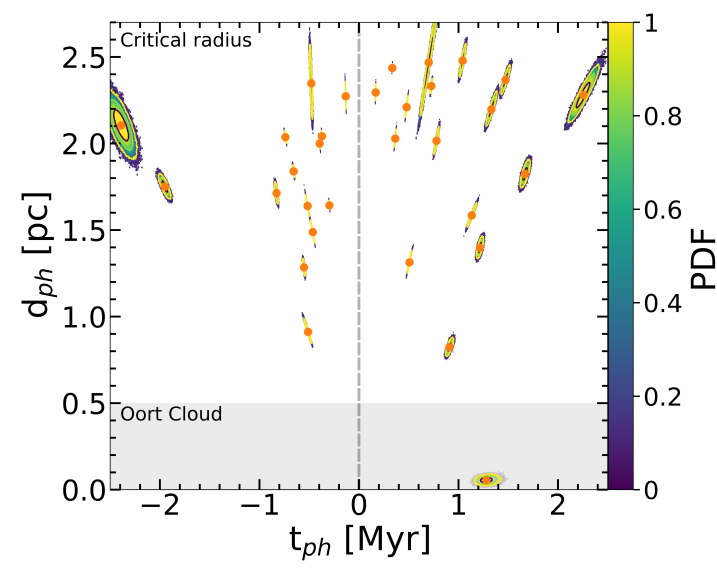

Fig. 4. Joint probability density of the time and distance of closest approach for those stars that are predicted to pass within $2.5 \mathrm{pc}$ of the Sun (listed in Table 3). The contour levels indicate regions enclosing $0.6,0.9$, and $0.99 \%$ cumulative probability (colour bar). The shape of each PDF is affected by the relative errors in the observational data of each star, particularly the errors on parallax and radial velocity.

distances, while as we showed in Sect. 3.2 the cumulative effect of passing stars can also lead to substantial perturbations of

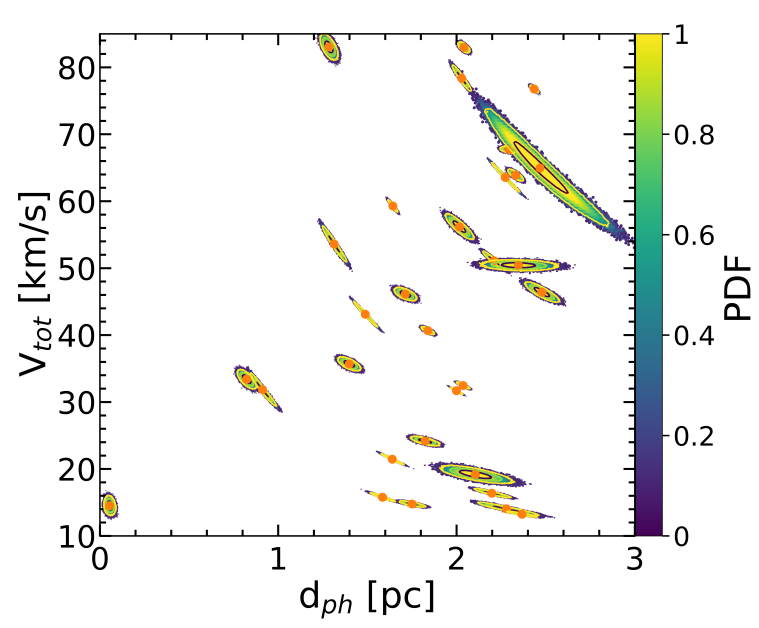

Fig. 5. Joint probability density of the time and relative velocity of closest approach for the stars in Table 3.

the Oort cloud comets. In this section we study the cumulative effects of the known stellar encounters (Table 4) and the Galactic tidal field over the interval of $20 \mathrm{Myr}$ centred on the present time. This provides a lower limit to the combined effect of 


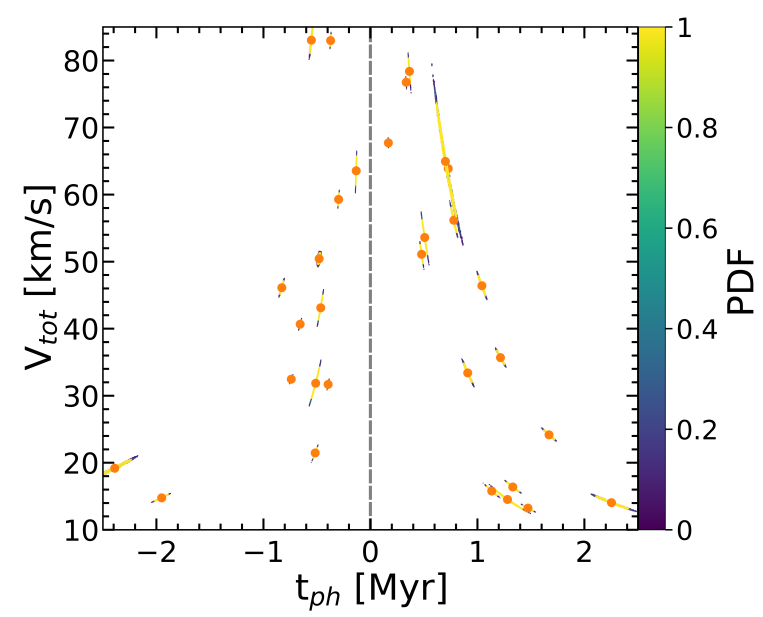

Fig. 6. Joint probability density of the distance and relative velocity of closest approach for the stars in Table 3.

stellar encounters and the Galactic tidal field on the dynamical evolution of the Oort cloud.

\subsection{Numerical model}

We use the Astrophysical Multi-purpose Software Environment (AMUSE; Portegies Zwart et al. 2009, 2013; Pelupessy et al. 2013; Portegies Zwart \& McMillan 2018) for our calculations. Following the works of Rickman et al. (2008) and Hanse et al. (2016) we first construct an isotropic Oort cloud of 10000 test particles (Fig. 9, first row). The distribution of Oort cloud particles is spherically symmetric and isotropic, and they follow a uniform distribution in the orbital elements $\cos i, \omega, \Omega$, and $M$. The initial eccentricities, $e$, are selected with a probability density distribution $p(e) \propto e$ and the perihelia, $q$, are chosen outside of the planetary region $(q>32 \mathrm{AU})$. The semi-major axes, $a$, are distributed proportional to $a^{-1.5}$ over the range $3 \times 10^{3}-10^{5}$ AU. In order to ensure a thermalised Oort cloud (e.g. Duncan et al. 1987; Dybczyński 2002; Rickman et al. 2008) we used a radial density profile of $r^{-3.5}$ (where $r$ is the distance between the comets and the Sun).

Subsequently we used the GPU-accelerated direct N-body code ABIE (Cai et al., in prep.) with a fifteenth-order Gauss-Radau integrator (Everhart 1985) optimised for close encounters. We couple ABIE and the Gala package in such a way that ABIE advances the positions of the Oort cloud particles and Gala calculates the accelerations on each particle due to the Galactic tidal field, based on the positions provided by ABIE. The calculated accelerations are subsequently inserted into the Gauss-Radau integrator in ABIE as additional forces. Using the catalogue of nearby stars (Table 3 ), we selected all the stars (31) that are predicted to pass within $2.5 \mathrm{pc}$ of the Sun (Table 4) \pm 10 Myr from today. These stars are included in the integrator with their present-day positions and velocities with respect to the Sun. Hence we evolve a system for a period of $20 \mathrm{Myr}$ which consists of one host star (the Sun) surrounded by 10000 test particles (Oort cloud) under the influence of external perturbations due to passing stars and the Galactic tidal field.

\subsection{Galactic tide and Gaia star perturbation}

In order to disentangle the effects of the Galactic tidal field and the encounters with stars identified in Gaia DR2 we considered three main cases for external perturbations, Galactic tidal field, Gaia stars, Galactic tidal field + Gaia stars. We focus now on the effect of the external perturbations considering an extended Oort cloud ( $a \leq 100000 \mathrm{AU}$ ). The first row of Fig. 9 shows the initial conditions followed by the final perihelion distance as a function of the final semi-major axis for the three scenarios previously discussed. Considering a short integration of $20 \mathrm{Myr}$ (10 Myr in the past, and $10 \mathrm{Myr}$ in the future). The green area represents the original location of the ejected particles (yellow dots).

The effect of the Galactic tidal field on the Oort cloud decreases from the outskirts to the inner regions of the cloud (second row Fig. 9). The particles at the edge of the cloud suffer a considerable change in their orbital elements. Specifically, for $\sim 0.91 \%$ (yellow dots, Fig. 9) of the objects, their semi-major axes increase up to interstellar distance ( $a>100000 \mathrm{AU})$. The particles in the inner Oort cloud remain unaffected. A small fraction of the particles $(\sim 0.02 \%)$ acquire hyperbolic orbits. When Gaia stars are the only perturber (Fig. 9, third row) their effect is much less pronounced than that of the Galactic tidal field in particular in the outskirts on the Oort cloud. The effect of Gaia stars is dominated by the star GJ 710 (Fig. 8). However, the cumulative effect of relatively distant encounters $(\sim 1 \mathrm{pc})$ helps to change the semi-major axis of $\sim 0.38 \%$ of the comets in the outer Oort cloud, whereas $0.01 \%$ of the outer Oort cloud objects acquire hyperbolic orbits.

The combination of the Galactic tidal field and Gaia stars (Fig. 9, last row) enhances the perturbations on the Oort cloud, causing $0.03 \%$ of the initial objects to become unbound from the solar system, while $\sim 1.12 \%$ acquire orbits with semi-major axis in the interstellar regions, i.e., $a \geq 100000 \mathrm{AU}$. In all three scenarios for external perturbations, a considerable population of objects with $a \geq 100000 \mathrm{AU}$ is created. Their orbits remain elliptic, but the effect of external perturbations lifts their semimajor axis beyond the Oort cloud (yellow dots, Fig. 9). This effect is only relevant for the outermost regions of the Oort cloud ( 80000-100 000 AU). The orbital elements of the particles in the inner parts of the cloud will not be affected as strongly.

We now consider a compact Oort cloud with semi-major axes up to $50000 \mathrm{AU}$. The effect of the Galactic tidal field has a negligible effect (first panel, Fig. 10) over the particles in the cloud. The second panel in Fig. 10 shows the effect of the Gaia stars. The effect of GJ 710 is prominent, and causes a major perturbation. The last panel in Fig. 10 shows that the effect of the Gaia stars dominates over the Galactic tidal field, however it is the combined effect which efficiently increases the number of particles (by $\sim 1.20 \%$ ) with semi-major axis beyond the limits of the cloud $(a>50000 \mathrm{AU})$.

We conclude that the cumulative effect of passing stars and the Galactic tidal field are efficient mechanisms in the creation of comets for which the semi-major axis is larger than the extent of the Oort cloud ( $a>100000 \mathrm{AU})$, but with bound and eccentric orbits. Hereafter, we refer to such objects as transitional interstellar comets (TICs). If we consider an Oort cloud with $a \leq 100000 \mathrm{AU}$, the Galactic tidal field is the major perturber, while for an Oort cloud with $a \leq 50000$ AU passing stars provide the major effect, mainly due to the close encounter with GJ 710 (Fig. 8).

For long timescales (on the order of gigayears), the synergy between Galactic tides and stellar encounters to bring comets into the observable zone is now well understood (Rickman et al. 2008; Fouchard et al. 2011). Both perturbations strongly depend on the semi-major axis of the comets. In general the Galactic tidal field rapidly changes the perihelia of the outer regions, while passing stars are a good mechanism to eject or inject 

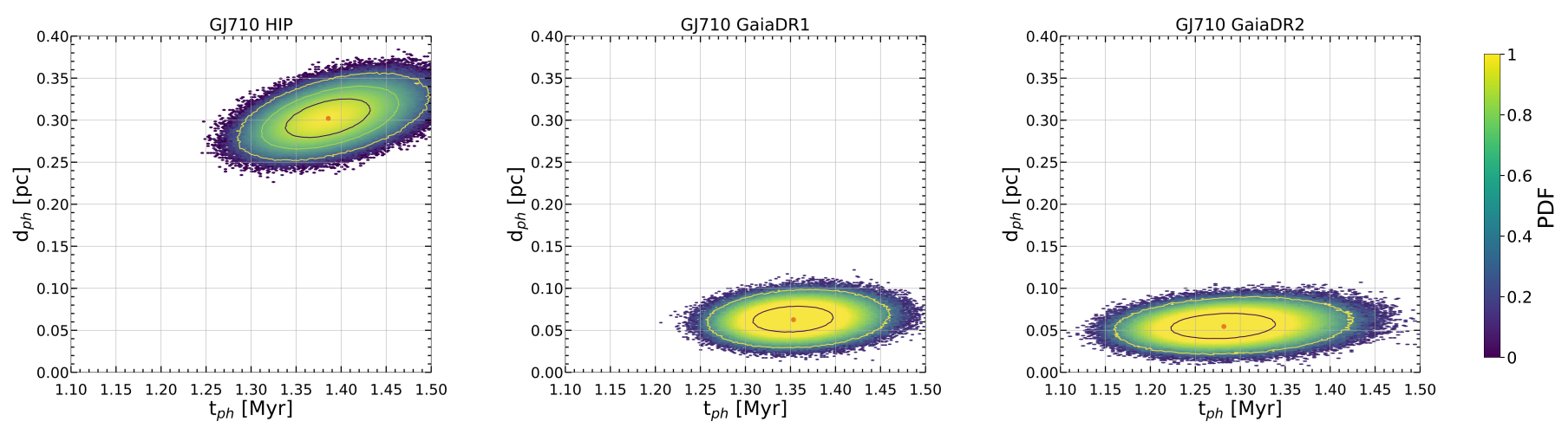

Fig. 7. Joint distribution of the time and distance of closest approach for GJ 710. Leftmost panel: results obtained with HIPPARCOS data, while middle and right panels: results for Gaia DR1 and Gaia DR2, respectively (see Table 5). The contour levels indicate regions enclosing 0.60, 0.90, and $0.99 \%$ cumulative probability (color bar).

Table 5. Comparison of the different astrometric parameters and radial velocities obtained for GJ 710 from HIPPARCOS, Gaia DR1, and Gaia DR2 data.

\begin{tabular}{lccc}
\hline \hline Parameters & HIPPARCOS & Gaia DR1 & Gaia DR2 \\
\hline$\varpi(\mathrm{mas})$ & $51.12 \pm 1.63$ & $52.35 \pm 0.27$ & $52.51 \pm 0.04$ \\
$\mu_{\alpha *}\left(\mathrm{mas} \mathrm{yr}^{-1}\right)$ & $1.15 \pm 1.66$ & $-0.47 \pm 0.13$ & $-0.45 \pm 0.08$ \\
$\mu_{\delta}\left(\mathrm{mas} \mathrm{yr}^{-1}\right)$ & $1.99 \pm 1.22$ & $-0.18 \pm 0.09$ & $-0.02 \pm 0.07$ \\
$\mu_{\mathrm{tot}}\left(\mathrm{mas} \mathrm{yr}^{-1}\right)$ & $2.30 \pm 2.06$ & $0.50 \pm 0.16$ & $0.46 \pm 0.11$ \\
$v_{\mathrm{rad}}\left(\mathrm{km} \mathrm{s}^{-1}\right)$ & $-13.80 \pm 0.30^{(*)}$ & $-13.80 \pm 0.30^{(*)}$ & $-14.52 \pm 0.43^{(* *)}$ \\
$t_{\mathrm{ph}}(\mathrm{Myr})$ & $1.385[1.109,1.500]$ & $1.353[1.219,1.541]$ & $1.281[1.109,1.500]$ \\
$d_{\mathrm{ph}}(\mathrm{pc})$ & $0.302[0.302,0.324]$ & $0.062[0.014,0.116]$ & $0.054[0.003,0.106]$ \\
\hline
\end{tabular}

Notes. The last two rows represents the time and the closest approach distance to the Sun of GJ 710 with its respective confidence interval.

References. ${ }^{(*)}$ Pulkovo catalogue (Gontcharov 2006), ${ }^{(* *)}$ Gaia DR2 radial velocity catalogue (Soubiran et al. 2018).

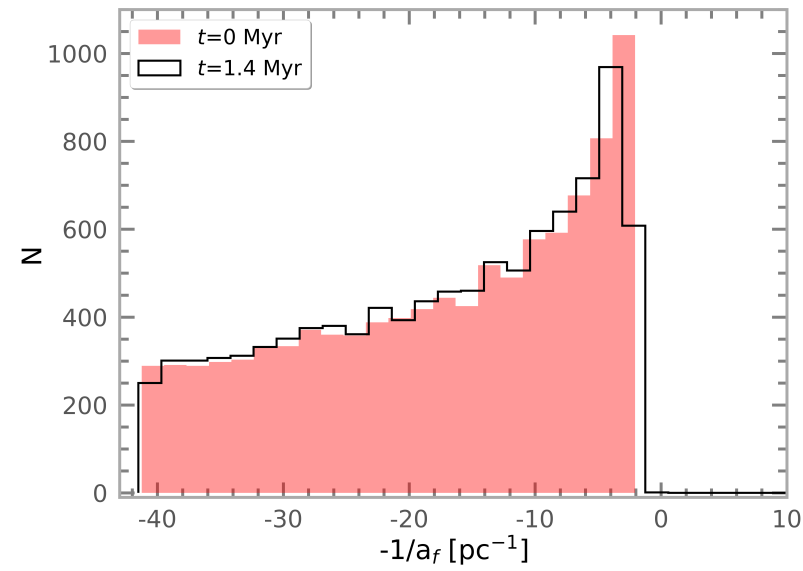

Fig. 8. Histogram of the orbital energy distribution of the particles in the Oort cloud, before and after the encounter with the star GJ710. The red histogram corresponds to the initial semi-major axis distribution, and the black curve to the final one.

particles when a close encounter happened (see e.g., Portegies Zwart \& Jílková 2015). For short timescales ( 20 Myr), the Galactic tide and stellar encounters prove to be an efficient mechanism for the creation of TICs. The outermost part of the cloud ( 80 000-100 $000 \mathrm{AU}$, Fig. 11) is heavily perturbed, whereas the innermost part remains unchanged (3000-50000 AU, Fig. 11). This implies that the edge of the Oort cloud is sensitive to external perturbations and is relatively easy to strip. The particles in the outermost part of the Oort cloud have a considerable change in their orbital elements. The change of the perihelion and eccentricity increases as a function of the semi-major axes (Fig. 12), whereas the semi-major axes reach interstellar distances. These objects previously referred to as transitional interstellar comets remain bound to the Sun with eccentric orbits (Fig. 13). The detailed effects of subsequent perturbations due to passing stars and the Galactic tidal filed will determine if these objects will return to the solar system or become unbound.

Considering the efficiency of external perturbations on circumstellar comet clouds in the creation of interstellar objects, and noting that Valtonen \& Innanen (1982) pointed out that objects with a relative velocity above $0.5 \mathrm{~km} \mathrm{~s}^{-1}$ can probably enter and leave the solar system, we speculate that a "cloud" of objects exists in interstellar space which overlaps with our Oort cloud and constantly exchanges material with it. An indication that this may be the case was provided by the first interstellar comet detected, 'Oumuamua (Williams 2017), which opened a new era in the study of interstellar objects. Estimates of the local density of interstellar objects range from $10^{14} \mathrm{pc}^{-3}$ (Portegies Zwart et al. 2018), to $8 \times 10^{14} \mathrm{pc}^{-3}$ (Jewitt et al. 2017), to $2 \times 10^{15} \mathrm{pc}^{-3}$ (Do et al. 2018). The existence of an interstellar comet cloud could partly explain the slightly hyperbolic comets and potential interstellar objects that might have been detected in the solar system but not yet classified as such (see e.g. Ashton et al. 2018; de la Fuente Marcos et al. 2018; Siraj \& Loeb 2019). A future detailed study of the evolution of the TICs created by the tides and stellar encounters is needed to draw more solid conclusions. 

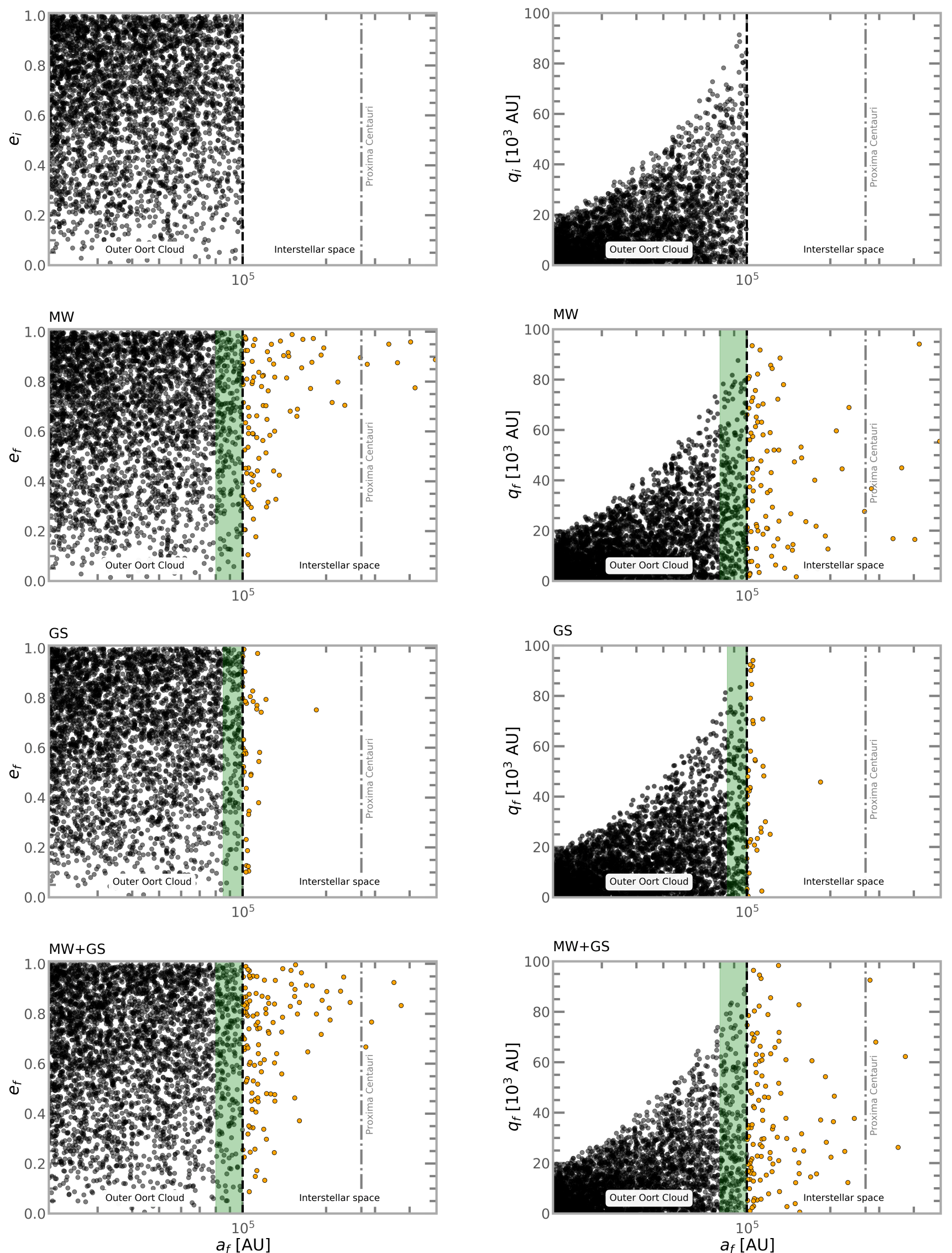

Fig. 9. Final eccentricity and perihelion as a function of final semi-major-axis, for a total integration of 20 Myr. First row: initial conditions. Second and third rows: effect of the Galactic tidal field and Gaia stars, respectively. Last row: combined effects of the Galactic tides and Gaia stars. The green area corresponds to the initial position of the ejected particles, coloured yellow. 

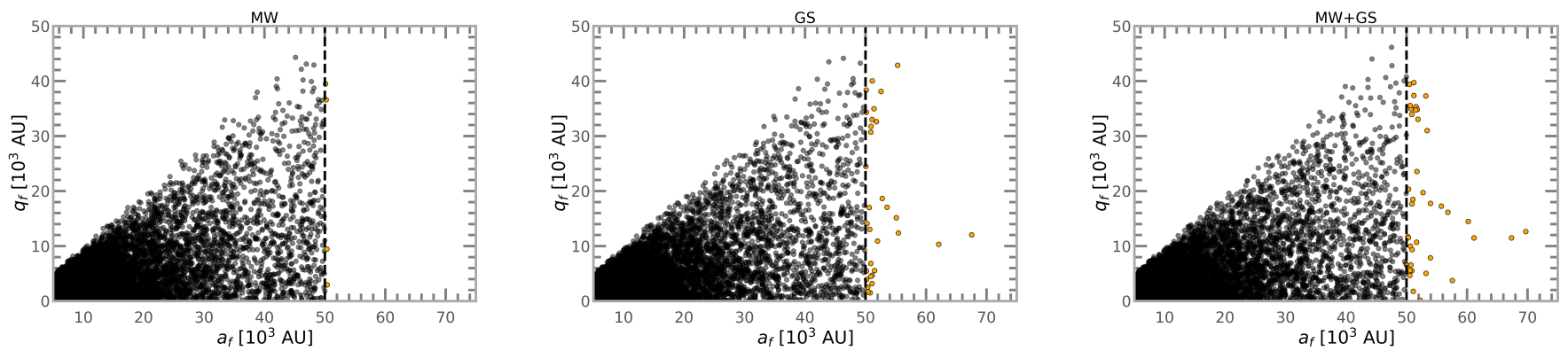

Fig. 10. Final perihelion as a function of final semi-major axis for an Oort cloud size of 50000 AU. First panel: effect of the Galactic tidal field (marked as a MW). Second and third panels: effect of Gaia stars (GS) and the combination of both the Galactic tide and Gaia stars (MW+GS), respectively. Black dots represent the particles in the Oort cloud, while the yellow dots represent the ejected ones.

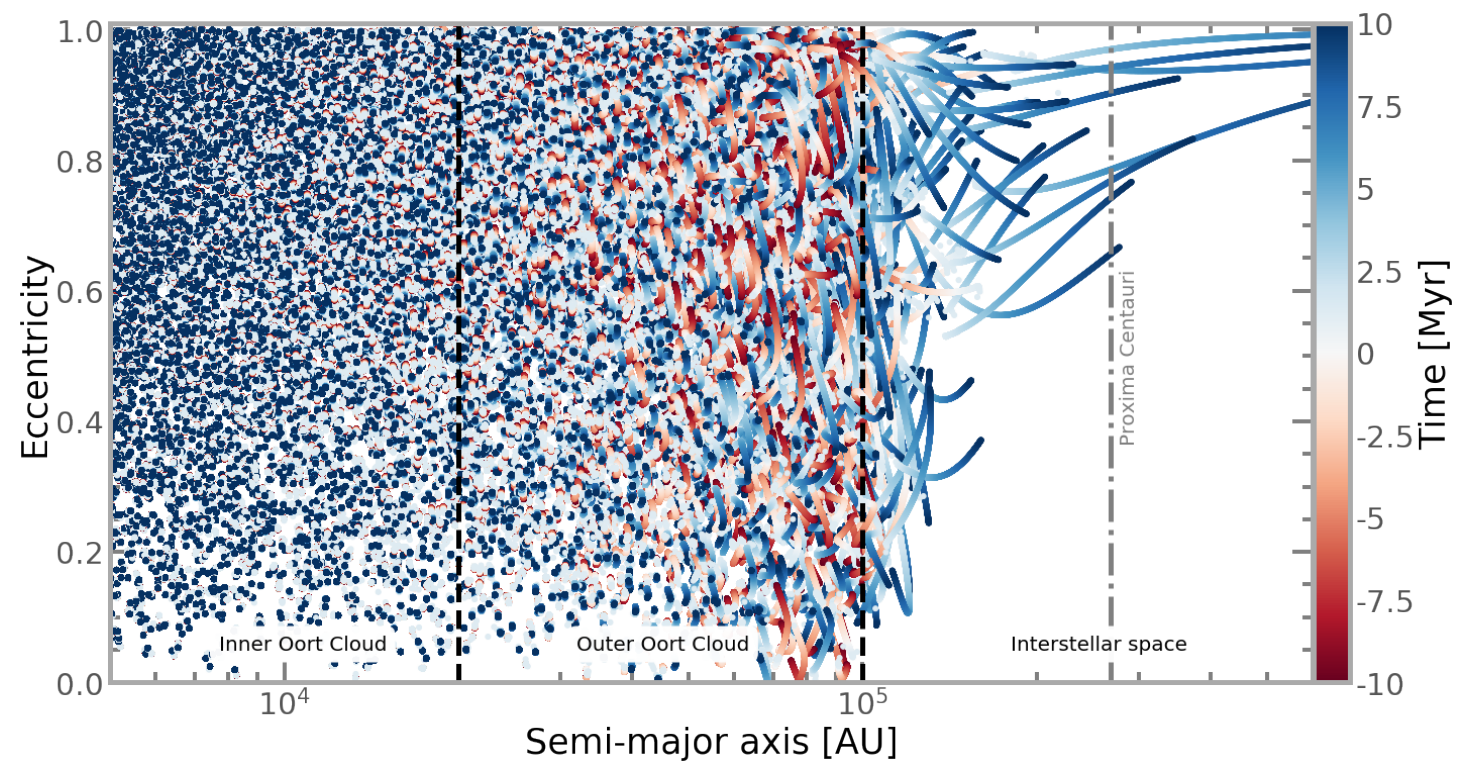

Fig. 11. Orbital evolution of the particles in the simulated Oort cloud. The figure shows the evolution of the eccentricity as a function of the semi-major axis over the period of $20 \mathrm{Myr}( \pm 10 \mathrm{Myrs}$.). The colour bar represents the integration time. An animation can be found online and at https://home.strw. leidenuniv.nl/ storres/\#Research.

The results presented here are based on the assumption of a hypothetical present day spheroidal cloud of comets extending up to $100000 \mathrm{AU}$ from the Sun. If we consider a smaller structure (Fig. 10), passing stars are the main perturbers, while the Galactic tidal field barely influences the orbit of the comets. In addition we stress that our sample of stars considered as perturbers of the Oort cloud is incomplete due to the Gaia survey limits combined with our data quality filtering and the upper limit we imposed on the distance to the stars in our sample. A more complete inventory of Oort cloud perturbers would increase the effects of the stellar encounters.

\section{Summary and conclusions}

In this work we present a study of the combined effect of the Galactic tidal field and close stellar encounters predicted to occur over a time interval of $20 \mathrm{Myr}$ around the present on the Oort cloud of the solar system. Our focus is on the loss of comets to interstellar space. Following Rickman et al. (2008), we first presented a simple model of stellar encounters based on data compiled for 13 spectral types of the stars in the solar neighbourhood. We confirm that individual perturbations of randomly passing stars cannot alter the orbits of the comets in the Oort cloud unless a very close encounter occurs. However, from a consideration of the stellar encounter statistics we show that the comets in the cloud may be lost to interstellar space over a short period of time due to the cumulative effect of stellar encounters.

Motivated by this result we used Gaia DR2 data to identify 14659 stars passing within $50 \mathrm{pc}$ of the Sun over the time period of \pm 10 Myr centred on the present. Out of this sample 31 stars are predicted to be major perturbers of the Oort cloud, approaching the Sun to within $2.5 \mathrm{pc}$. This catalogue of perturbing stars (presented in Table 3) constitutes an astrometrically clean sample, which is nevertheless incomplete due to the Gaia survey limitations, the upper limit imposed on the distance to the stars in the sample $(50 \mathrm{pc})$, and the strict data-quality filtering. Our estimates of the effect of known stellar encounters is therefore conservative (we note that Bailer-Jones et al. 2018, find a larger number of stellar encounters from Gaia DR2 due to their less stringent dataquality filtering). Using the impulse approximation (Eq. (1)) we then calculated the impulse that each star passing within $2.5 \mathrm{pc}$ of the Sun imparts to a comet in the Oort cloud. We found that (as expected) the effect of individual encounters is relatively small (on the order of $10^{-3}$ to $10^{-4} \mathrm{~km} \mathrm{~s}^{-1}$ ). The cumulative effect of Gaia stars was then investigated. We found that the collective effect of stars passing within $\sim 1$ pc can lift the perihelion of members of the Oort cloud in a relatively short period of time. 

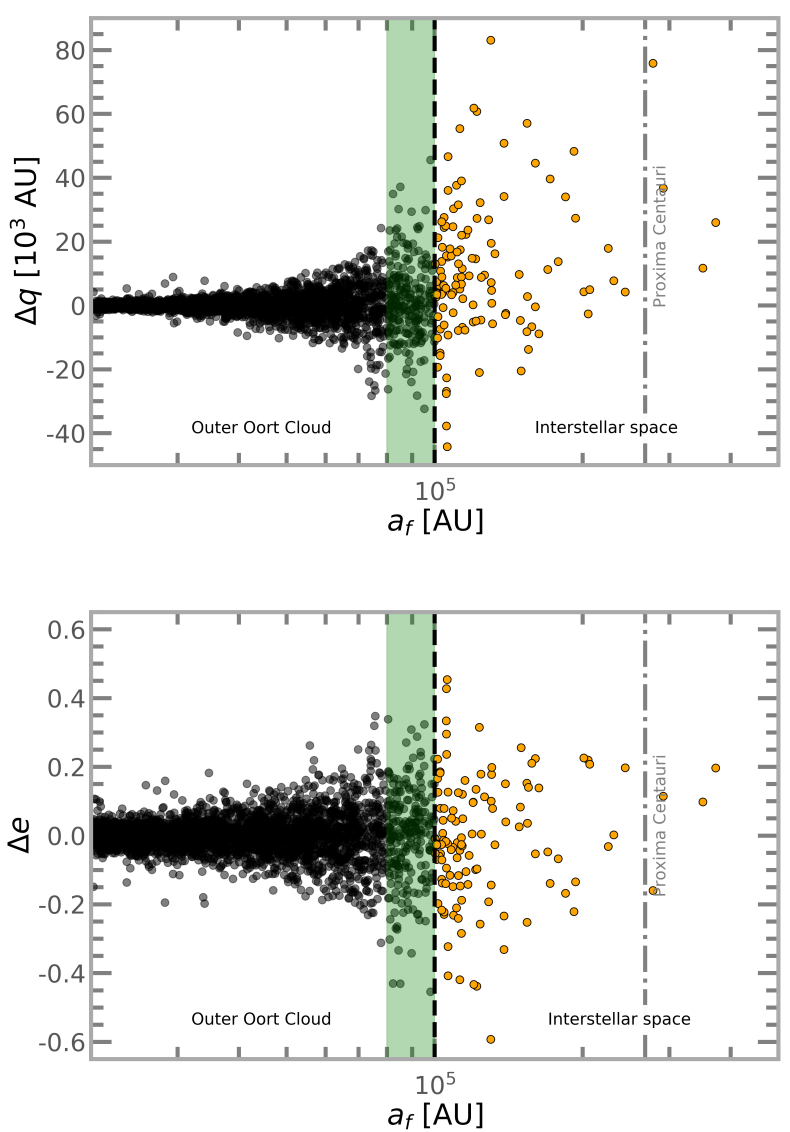

Fig. 12. Mean perihelion and eccentricity changes as a function of the semi-major axis of the comets. The green area corresponds to the region of the initial position of the particles ejected, represented as yellow dots.

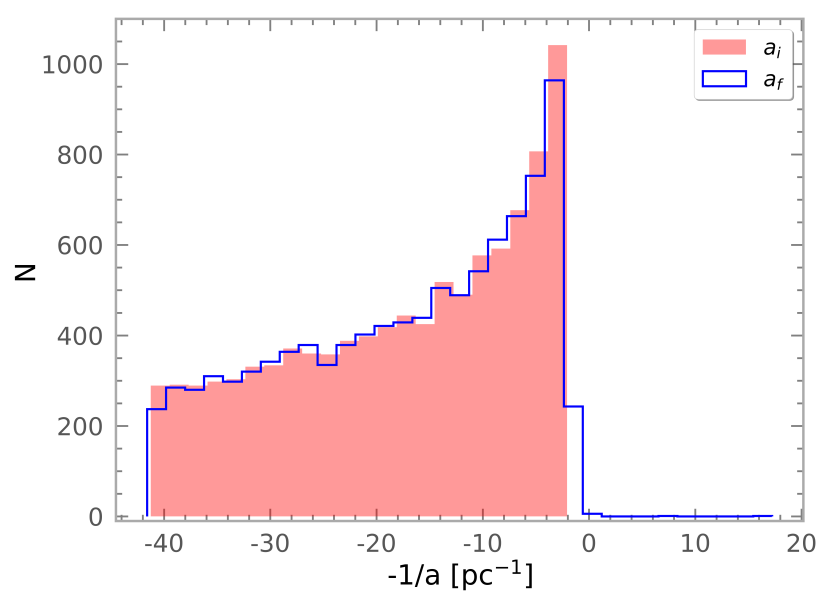

Fig. 13. Histogram of the orbital energy distribution of the particles in the Oort cloud. The blue curve corresponds to the final semi-major axis, while the red curve shows the initial distribution.

Finally, we focused our study on the combined effect of multiple stellar encounters and the Galactic tidal field on a simulated Oort cloud. To achieve this we used a detailed $N$-body simulations, evolving a system of one host star (the Sun) surrounded by 10000 test particles (the Oort cloud) and affected by three different sets of external perturbations (Gaia stars only, Galactic tidal field only, and the combination of both), over a period of $20 \mathrm{Myr}$ ( \pm 10 Myr centred on today). When we consider an extended Oort cloud ( $a \leq 100000 \mathrm{AU})$, we find that the effect of the Galactic tidal field alone leads to the creation of TICs of around $0.91 \%$ of the initial comets, while the collective effect of the passing stars only leads to a smaller fraction of $0.38 \%$. For the compact model of the Oort cloud ( $a \leq 50000 \mathrm{AU})$, passing stars dominate the perturbations, mainly due to the star GJ 710, while the effect of the Galactic tidal field is almost negligible. Overall for an extended cloud, the Galactic tide dominates over the passing stars, for the case of a compact cloud the opposite is true. However, it is the combined effect of passing stars and the Galactic tidal field which significantly increases the perturbation on the Oort cloud. These combined effects raise the semi-major axis of around $1.12 \%$ of the initial particles for the compact model, and $\sim 1.20 \%$ for the extended one, up to the interstellar regions (i.e. $a>100000 \mathrm{AU})$. The estimates presented in this work are conservative and based on a small sample of known stars that pass near to the Sun during \pm 10 Myr. The effects of a more complete sample will increase the number of TICs. Overall the external perturbations are an efficient mechanisms in the formation of interstellar comets over a short period of time (in the order of tens of megayears).

The further evolution of the transitional interstellar comets depends on the perturbations introduced by passing stars and the Galactic tidal field. These perturbations determine whether the transitional interstellar comets will remain bound to the solar system or eventually become interstellar comets. Under the hypothesis that other planetary systems also possess Oort cloud-like structures, they most probably experience the same mechanism of erosion due to external perturbations. This leads us to speculate that there is a large population of cometary bodies that occupy interstellar space. Therefore, visits to the solar system by interstellar comets such as 'Oumuamua may well be a frequent occurrence.

Acknowledgements. We thank the referee for the constructive reports and helpful suggestions to improve the present work. ST expresses his gratitude to the Mexican National Council for Science and Technology (CONACYT) for the grant \#291004-410 780; to Leiden Observatory for the unconditional support; and to Daniel Hestroffer, Eric Mamajek, Konstantin Batygin, and Ylva Götberg, for the discussions and comments on the manuscript. This project was shaped in part at the 2018 New York Gaia Sprint. This work was supported by the Netherlands Research School for Astronomy (NOVA) and by NWO (grant \#621.016.701 [LGM-II]). This work has made use of data from the European Space Agency (ESA) mission Gaia (https://www.cosmos.esa.int/gaia), processed by the Gaia Data Processing and Analysis Consortium (DPAC, https://www. cosmos.esa.int/web/gaia/dpac/consortium). Funding for the DPAC has been provided by national institutions, in particular the institutions participating in the Gaia Multilateral Agreement. This work was carried out on the Dutch national e-infrastructure with the use of Cartesius the Dutch national supercomputer and the support of SURF Cooperative.

\section{References}

Abolfathi, B., Aguado, D. S., Aguilar, G., et al. 2018, ApJS, 235, 42 Anderson, E., \& Francis, C. 2012, Astron. Lett., 38, 331

Andrae, R., Fouesneau, M., Creevey, O., et al. 2018, A\&A, 616, A8

Ashton, E., Gladman, B., Kavelaars, J., \& Williams, G. 2018, AAS/Div. Planet. Sci. Meeting Abstracts, 50, 201.02

Astropy Collaboration (Price-Whelan, A. M., et al.) 2018, AJ, 156, 123

Bailer-Jones, C. A. L. 2015, A\&A, 575, A35

Bailer-Jones, C. A. L. 2018, A\&A, 609, A8

Bailer-Jones, C. A. L., Rybizki, J., Andrae, R., \& Fouesneau, M. 2018, A\&A, 156, A58

Berski, F., \& Dybczyński, P. A. 2016, A\&A, 15, A4

Bobylev, V. V., \& Bajkova, A. T. 2017, Astron. Lett., 43, 559

Bovy, J. 2015, ApJS, 216, 29

Bovy, J. 2017, MNRAS, 1387, 1360

Brasser, R., \& Morbidelli, A. 2013, Icarus, 225, 40

Brasser, R., Duncan, M. J., \& Levison, H. F. 2006, Icarus, 184, 59

Buder, S., Asplund, M., Duong, L., et al. 2018, MNRAS, 478, 4513

de la Fuente Marcos, R., \& de la Fuente Marcos, C. 2018, Res. Notes AAS, 2, 30 
de la Fuente Marcos, C., de la Fuente Marcos, R., \& Aarseth, S. J. 2018, MNRAS, 476, L1

Dehnen, W., \& Binney, J. J. 1998, MNRAS, 298, 387

Do, A., Tucker, M. A., \& Tonry, J. 2018, ApJ, 855, L10

Dones, L., Weissman, P. R., Levison, H. F., \& Duncan, M. J. 2004, Comets II (Tucson, AZ: University of Arizona Press), 323, 153

Dones, L., Brasser, R., Kaib, N., \& Rickman, H. 2015, Space Sci. Rev., 197, 191

Duncan, M. J. 2008, Space Sci. Rev., 138, 109

Duncan, M. J. T. Q., \& S. T. 1987, AJ, 94, 1330

Dybczyński, P. A. 2002, Earth, Moon Planets, 90, 483

Dybczyński, P. A., \& Berski, F. 2015, MNRAS, 2471, 13

Everhart, E. 1985, Dynamics of Comets: Their Origin and Evolution, IAU Colloq. 83, 115

Feng, F., \& Bailer-Jones, C. A. 2015, MNRAS, 454, 3267

Fouchard, M., Froeschlé, C., Valsecchi, G., \& Rickman, H. 2006, Celest. Mech. Dyn. Astron., 95, 299

Fouchard, M., Rickman, H., Froeschle, C., \& Valsecchi, G. B. 2011, A\&A, 535, A86

Fouchard, M., Higuchi, A., Ito, T., \& Maquet, L. 2018, A\&A, 45, A1

Gaia Collaboration (Brown, A. G. A., et al.) 2016a, A\&A, 595, A2

Gaia Collaboration (Prusti, T., et al.) 2016b, A\&A, 595, A1

Gaia Collaboration (Brown, A. G. A., et al.) 2018, A\&A, 616, A1

García-Sánchez, J., Preston, R. A., Jones, D. L., et al. 1999, AJ, 117, 1042

García-Sánchez, J., Weissman, P. R., Preston, R. A., et al. 2001, A\&A, 379, 634

Gontcharov, G. A. 2006, Astron. Lett., 32, 759

Hanse, J., Jílková, L., Portegies Zwart, S. F., \& Pelupessy, F. I. 2016, MNRAS, 473,5432

Heisler, J., \& Tremaine, S. 1986, Icarus, 65, 13

Hernquist, L. 1990, AJ, 359

Higuchi, A., \& Kokubo, E. 2015, AJ, 150, 26

Hills, J. 1981, AJ, 86, 1730

Jewitt, D., Luu, J., Rajagopal, J., et al. 2017, ApJ, 850, L36

Jílková, L., Portegies Zwart, S., Pijloo, T., \& Hammer, M. 2015, MNRAS, 453, 3158

Jílková, L., Hamers, A. S., Hammer, M., \& Zwart, S. P. 2016, MNRAS, 457, 4218

Jiménez-Esteban, F. M., Torres, S., Rebassa-Mansergas, A., et al. 2018, MNRAS, 4518,4505

Jimenez-Torres, J. J., Pichardo, B., Lake, G., \& Throop, H. 2011, MNRAS, 418, 1272

Kaib, N. A., \& Quinn, T. 2008, Icarus, 197, 221

Kunder, A., Kordopatis, G., Steinmetz, M., et al. 2017, AJ, 153, 75

Levison, H. F., Morbidelli, A., \& Dones, L. 2004, AJ, 128, 2553

Lindegren, L., Hernández, J., Bombrun, A., et al. 2018, A\&A, 616, A2
Mamajek, E. E., Barenfeld, S. A., Ivanov, V. D., et al. 2015, ApJ, 800, L17 Martínez-Barbosa, C. A., Brown, A. G. A., Boekholt, T., et al. 2016, MNRAS, 457, 1062

Martínez-Barbosa, C. A., Jílková, L., Portegies Zwart, S., \& Brown, A. G. 2017, MNRAS, 464, 2290

Matthews, R. A. J. 1994, QJRAS, 35, 1

Miyamoto, M., \& Nagai, R. 1975, Astron. Soc. Jpn., 27, 533

Morbidelli, A. 2008, 1

Navarro, J. F., Frenk, C. S., \& White, S. D. M. 1996, AJ, 462, 563

Oort, J. H. 1950, Communications from the Observatory of Leiden, 408

Pecaut, M. J., \& Mamajek, E. E. 2013, ApJS, 208, 9

Pelupessy, F. I., van Elteren, A., de Vries, N., et al. 2013, A\&A, 557, A84

Perryman, M. A. C., Lindegren, L., Kovalevsky, J., et al. 1997, A\&A, 323, L49

Portegies Zwart, S., \& Jílková, L. 2015, MNRAS, 451, 144

Portegies Zwart, S., \& McMillan, S. 2018, Astrophysical Recipes: The Art of AMUSE (Bristol: Iop Publishing Ltd)

Portegies Zwart, S., McMillan, S., Harfst, S., et al. 2009, New Astron., 14, 369

Portegies Zwart, S. F., Mcmillan, S. L. W., Elteren, A. V., Pelupessy, F. I., \& Vries, N. D. 2013, Comput. Phys. Commun., 184, 456

Portegies Zwart, S., Torres, S., Pelupessy, I., Bédorf, J., \& Cai, M. X. 2018, MNRAS, 479, L17

Price-Whelan, M. A. 2017, J. Open Source Softw., 2, 388

Reid, M. J., Menten, K. M., Brunthaler, A., et al. 2014, ApJ, 783, 130

Rickman, H. 1976, Bull. Astr. Inst. Czechosl., 27, 92

Rickman, H., Froeschlé, C., Froeschlé, C., \& Valsecchi, G. B. 2004, A\&A, 428, 673

Rickman, H., Fouchard, M., Froeschlé, C., \& Valsecchi, G. B. 2008, Celest. Mech. Dyn. Astron., 102, 111

Scholz, R.-D. 2014, A\&A, 561, A113

Schönrich, R., Binney, J., \& Dehnen, W. 2010, MNRAS, 403, 1829

Shannon, A., Jackson, A. P., Veras, D., \& Wyatt, M. 2014, MNRAS, 446, 2059

Siraj, A., \& Loeb, A. 2019, ApJ, 872, L10

Soubiran, C., Jasniewicz, G., Chemin, L., et al. 2018, A\&A, 616, A7

Torres, S., Zwart, S. P., \& Brown, A. G. A. 2018, Proc. Int. Astron. Union, 12, 269

Valtonen, M. J., \& Innanen, K. A. 1982, AJ, 307

Veras, D., Evans, N. W., Wyatt, M. C., \& Tout, C. A. 2013, MNRAS, 437, 1127

Veras, D., Shannon, A., \& Boris, T. G. 2014, MNRAS, 445, 4175

Weissman, P. R. 1996, Earth, Moon Planets, 72, 25

Wiegert, P., \& Tremaine, S. 1999, Icarus, 137, 84

Williams, G. 2017, Minor Planet Electron. Circ., 2017-U181

Zhao, G., Zhao, Y. H., Chu, Y. Q., Jing, Y. P., \& Deng, L. C. 2012, Res. A\&A, 12,723 